\title{
Análise do uso de mensagens de celular na melhoria da participação de estudantes em cursos a distância: um estudo de caso
}

Edgar Marçal, Universidade Federal do Ceará, edgar@virtual.ufc.br Rossana Andrade, Universidade Federal do Ceará, rossana@ufc.br Windson Viana, Universidade Federal do Ceará, windson@virtual.ufc.br Eduardo Junqueira, Universidade Federal do Ceará, eduardoj@virtual.ufc.br Rosemeiry Melo, Universidade Federal do Ceará, rmelo@ufc.br

Resumo. Estudos têm mostrado os benefícios das mensagens de celular na educação. Pesquisadores argumentam que estudos devem ser conduzidos para se avaliar a extensão dos benefícios e melhor identificar em quais contextos as mensagens móveis são aplicadas da melhor forma. Nesse sentido, esse artigo examinou a utilização das mensagens SMS em um curso a distância com 474 alunos. O principal objetivo foi descobrir se o uso desta tecnologia poderia melhorar a participação dos estudantes no curso. Os estudantes foram divididos em dois grupos e os resultados mostram que os alunos que receberam as mensagens móveis tiveram resultados significativamente superiores aqueles que não receberam, sendo quase $20 \%$ mais efetivos com relação ao cumprimento das atividades e até 78 horas mais rápidos para concluí-las.

Palavras-chave: Mobile Learning, Mensagens de Celular e E-Learning.

\section{Analysis of the use of mobile messages in the improvement of the participation of students in distance courses: a case study}

\begin{abstract}
Studies have shown the benefits of mobile messages in education. Researchers argue that more studies must be conducted to assess the extension of these benefits, and to better identify in which context mobile messaging is the best approach to be applied. In this sense, our study examined a post-graduate class consisting of 474 learners in which Short Message Service was used. The main goal was to find out if this technology would improve student participation in the course. Two groups of learners were created and the results showed that students who received mobile messaging outperformed significantly students than did not receive. They were almost $20 \%$ more effective at completing their assigned activity and up to 78 hours faster than students from the control group.
\end{abstract}

Keywords: Mobile Learning, Mobile Phone Messages and E-Learning. 


\section{INTRODUÇÃ̃O}

Os dispositivos móveis (como smartphones e tablets) têm mudado a forma como as pessoas se comunicam. Diferentes ferramentas de comunicação sem fio estão disponíveis nesses dispositivos, as quais têm os transformados nos principais meios de conversação entre as pessoas. As empresas também têm utilizado os smartphones como mecanismo para se alcançar os clientes, por exemplo, através de mensagens móveis para informar sobre transações bancárias, pedidos de compra realizados ou avisos escolares (MEF, 2016).

A introdução dos dispositivos móveis na educação dá origem ao conceito de Mobile Learning (ou m-learning). Este paradigma proporciona aos estudantes o senso de liberdade para estudar em qualquer lugar e a qualquer momento através de conteúdos multimídia (como áudios, imagens e vídeos), que estão associados ao aspecto lúdico da aprendizagem. Os dispositivos móveis têm sido usados em diferentes áreas da educação, incluindo ciências para crianças (Hwang et al., 2011), conteúdo de matemática do ensino médio (Abu-al-aish et al., 2012), e cursos de computação na universidade (Boticki et al., 2013).

Uma das principais ferramentas dos smartphones utilizadas na educação são as mensagens móveis. Estudos têm demonstrado as vantagens de utilizá-las nos processos de ensino e aprendizagem, por exemplo: fixação do conteúdo ensinado em sala de aula; lembrete das tarefas que precisam ser executadas ou do conteúdo a ser estudado; troca de informações com colegas de sala; e, recebimento de material didático de apoio em tempo real (Petrova, 2010; Gasaymeh e Aldalalah, 2013; Plana et al., 2013). Apesar destes benefícios, o uso das mensagens móveis não apresenta resultados positivos em todas as situações, com problemas específicos apontados em alguns estudos, como custos de transmissão e segurança (Petrova, 2010; Plana et al., 2013).

Nesse sentido, este artigo apresenta um estudo de caso sobre o uso das mensagens móveis como recurso complementar no contexto de um curso de especialização à distância. Com isso, pretende-se investigar se as mensagens de celular podem melhorar a participação dos alunos no curso, considerando tanto o critério da conclusão das atividades quanto do tempo em que elas são realizadas. Além disso, busca-se avaliar a aceitação dos estudantes participantes sobre o uso desta tecnologia.

\section{TRABALHOS RELACIONADOS}

O Fórum de Ecossistema Móvel (em inglês Mobile Ecosystem Forum - MEF) conduziu um estudo com aproximadamente 6.000 participantes de 9 países para investigar a importância das mensagens móveis para as pessoas e empresas (MEF, 2016). A Figura 1 mostra um gráfico indicando as principais instituições que utilizam as mensagens de celular para alcançar seus clientes. Além disso, o gráfico também mostra qual o tipo de tecnologia usada, se via serviço de mensagens de texto ou através de aplicativo de mensagem específico (chat app). Por exemplo, o estudo aponta que $33 \%$ das pessoas receberam mensagens SMS dos seus bancos, contra $22 \%$ que receberam uma mensagem via chat app dessas instituições financeiras.

A expansão dos aplicativos de mensagem via Internet está diminuindo a importância do serviço SMS. Entretanto, estudos justificam a relevância do uso das mensagens SMS quando se considera a comunicação entre as instituições (como empresas, universidades e órgãos do governo) e seus clientes (Church e de Oliveira, 2013; Guerena, 2016; Malcolm, 2016). Esse modo de comunicação é conhecido como A2P (application-to-person). De acordo com o estudo do MEF (2016), as instituições preferem usar mensagens SMS a um aplicativo de mensagem porque elas são mais ubíquas e não dependem de uma aplicação específica, nem de conexão com a Internet.

V. $14 \mathrm{~N}^{\mathrm{o}} 2$, dezembro, 2016 

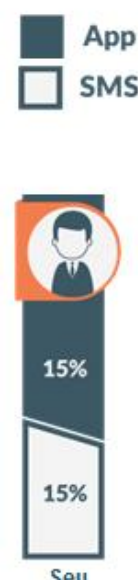

Seu empregador
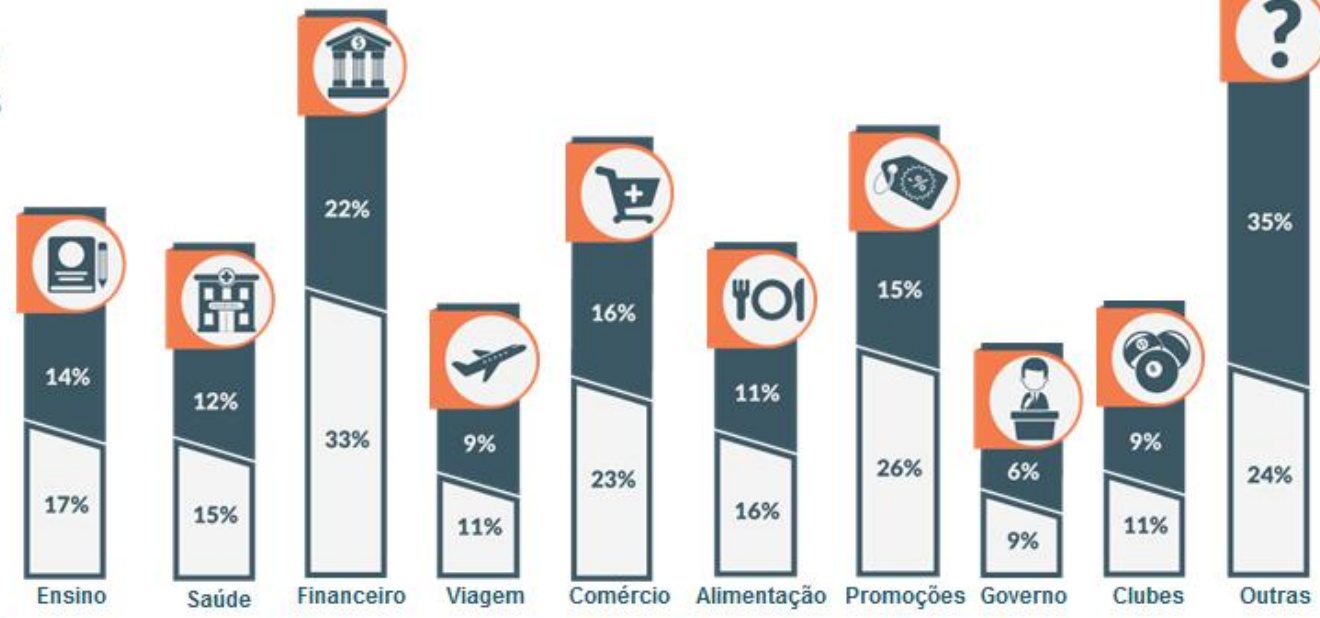

Figura 1 - Principais setores que utilizam as mensagens móveis (via SMS e chat app) (MEF, 2016)

Adicionalmente, o serviço de mensagem de texto está disponível em todos os celulares atuais, diferentemente dos chat app, que precisam ser instalados pelos usuários. Além disso, nesse caso, tanto o emissor quanto o receptor precisam ter $\mathrm{o}$ mesmo aplicativo (e.g., Whatsapp, Facebook, Telegram). Para instalar o chat app o usuário precisa realizar operações como efetuar configurações específicas, entrar com as informações de acesso (usuário e senha) e, dependendo da segurança do dispositivo, fornecer permissão para aplicação poder enviar e receber mensagens. Diferentemente, o serviço SMS já vem instalado e configurado de fábrica. Isso fornece um caráter de neutralidade ao serviço SMS, quando comparado aos aplicativos de mensagem, além de ser mais fácil para usuários menos experientes com as novas tecnologias. Além disso, Church e Oliveira apresentam um estudo que mostra que as pessoas dão mais importância às mensagens recebidas por SMS que aquelas via WhatsApp (Church e de Oliveira, 2013). Segundo os autores, isso acontece porque o SMS é um serviço pago e mais maduro.

A capacidade de receber e enviar mensagem com conteúdo educacional é uma das características mais importantes do paradigma Mobile Learning, oferecendo aos professores e estudantes uma possibilidade de aprendizagem mais flexível. Os objetivos do m-learning são: incrementar os recursos para aprendizagem dos estudantes; disponibilizar material didático em qualquer lugar e a qualquer momento; promover a aprendizagem formal e não formal; expandir os limites da sala de aula; e, favorecer o desenvolvimento de métodos inovadores de ensino através do uso das tecnologias móveis e sem fio (Marçal et al., 2005).

Diferentes benefícios podem ser obtidos através das ferramentas da computação móvel em prol da aprendizagem, por exemplo: mobilidade para usar os recursos computacionais fora de ambientes escolares tradicionais, novas oportunidades de aquisição de conteúdo, incentivo ao aluno para desenvolver habilidades em situações reais e facilidade para compartilhar e acessar remotamente material didático. No caso específico das mensagens móveis, elas destacam-se por maximizar a comunicação entre professores, alunos e a instituição de ensino (Chuang e Tsao, 2013; Hayati et al., 2013). Em (Petrova, 2010), é apresentado um estudo que avalia o impacto do uso de SMS entre universitários da Universidade da Nova Zelândia. Sobre o texto da mensagem, o estudo concluiu que: o conteúdo transmitido deve ser condensado e relevante para a aprendizagem dos alunos; e deve basear-se em alguma atividade concreta. $\mathrm{O}$ custo da transmissão das mensagens (dos alunos para Universidade) foi indicado como um 
problema, que inibiu a interação com o sistema. Os autores afirmam que as mensagens SMS representam uma forma interessante para implementar m-learning porque: (1) os estudantes podem obter conteúdo educacional através das mensagens, sem custos para recebê-las; (2) todos os tipos de celulares disponíveis atualmente suportam esse serviço; e (3) material adequado e personalizado é entregue ao estudante em tempo real.

Outros estudos mostram os benefícios do uso das mensagens móveis para educação através de aplicativos de mensagens como o WhatsApp e o KakaoTalk (Plana et al., 2013; Kim et al., 2014). Eles destacam que essas aplicações fornecem um ambiente virtual agradável para os alunos e que promovem a aquisição de conhecimento através da interação com colegas. Entretanto, alguns problemas identificados foram: segurança da informação trocada entre os usuários (Bere, 2013); dependência de conexão com a Internet (Kim et al., 2014); e dispersão dos estudantes que utilizam a aplicação para conversar com outras pessoas sobre outros assuntos (Plana et al., 2013).

A partir da análise dos trabalhos relacionados citados, identificamos os seguintes benefícios que podem ser alcançados através do uso das mensagens móveis nos processos de ensino e aprendizagem:

- As mensagens recebidas auxiliam na fixação do conteúdo lecionado em sala de aula;

- As mensagens móveis constituem um meio rápido e fácil de enviar e receber conteúdo;

- As mensagens móveis funcionam como um serviço de lembrete que avisa os estudantes sobre o que eles precisam estudar e quais tarefas precisam concluir;

- O uso de mensagens móveis é uma tecnologia familiar para maioria dos alunos;

- A facilidade de uso estimula a interação e troca de mensagens entre os estudantes;

- As questões e tarefas enviadas através das mensagens móveis mantêm os estudantes conectados ao curso.

Apesar das vantagens proporcionadas pelas mensagens móveis na educação, existem obstáculos que restringem seu uso em determinados contextos. A Tabela 1 mostra problemas encontrados com o uso dos dois tipos de mensagens móveis (via SMS ou chat app) e sugestões de solução através da outra forma de transmissão. A escolha do tipo de mensagem móvel a ser utilizado irá variar de acordo com a situação e deve levar em conta fatores como a disponibilidade de conexão de Internet, as habilidades do usuário, o tipo e tamanho das mensagens e o aporte de recursos financeiro disponível. Dependendo da situação, uma estratégia interessante é combinar as duas formas de envio de mensagens (SMS e aplicativo de mensagem).

Tabela 1 - Problemas e soluções do uso das mensagens móveis na educação

\begin{tabular}{ll}
\hline \multicolumn{1}{c}{ Problemas com Mensagens SMS } & \multicolumn{1}{c}{ Soluções via Aplicativos de Mensagens } \\
\hline $\begin{array}{l}\text { A quantidade limitada de caracteres por } \\
\text { mensagem é um fator limitante }\end{array}$ & $\begin{array}{l}\text { As mensagens dos chat app não têm limite de } \\
\text { tamanho. }\end{array}$ \\
\hline $\begin{array}{l}\text { As mensagens SMS não permitem o envio de } \\
\text { conteúdo multimídia (como imagens e áudios) }\end{array}$ & $\begin{array}{l}\text { Os aplicativos de mensagens suportam o } \\
\text { envio de diferentes conteúdos multimídia }\end{array}$ \\
\hline \multicolumn{1}{c}{ Problemas com Aplicativos de Mensagens } & \multicolumn{1}{c}{ Soluções via Mensagens SMS } \\
\hline $\begin{array}{l}\text { Necessidade de conexão e preocupação com a } \\
\text { segurança dos dados trafegados na Internet }\end{array}$ & $\begin{array}{l}\text { O serviço SMS não necessita de conexão } \\
\text { com a Internet. }\end{array}$ \\
\hline $\begin{array}{l}\text { O usuário precisa instalar e configurar no telefone } \\
\text { celular cada aplicativo de mensagem que for usar }\end{array}$ & $\begin{array}{l}\text { O celular já vem de fábrica pronto para } \\
\text { enviar e receber mensagens SMS }\end{array}$ \\
\hline $\begin{array}{l}\text { Os alunos se dispersão conversando com outras } \\
\text { pessoas sobre assuntos não escolares }\end{array}$ & $\begin{array}{l}\text { O perfil de uso do SMS é mais focado e } \\
\text { normalmente envolve uma quantidade menor } \\
\text { de mensagens e mais objetivas }\end{array}$ \\
\hline
\end{tabular}

V. $14 \mathrm{~N}^{\mathrm{o}} 2$, dezembro, 2016 


\section{METODOLOGIA}

Esta seção mostra como ocorreu o estudo de caso apresentado neste artigo, descrevendo o perfil dos participantes, as características das ferramentas utilizadas e os passos para realização do experimento.

\subsection{Participantes}

Esta pesquisa apresenta um experimento usando telefones celulares no curso de especialização semipresencial em Coordenação Pedagógica da Universidade Federal do Ceará realizado em 2013 para professores de escolas públicas. Os 474 alunos foram divididos em 10 turmas, cada uma contendo cerca de 40 alunos. Duas dessas turmas estavam localizadas na capital do Ceará e as outras oito em cidades do interior.

$\mathrm{O}$ perfil dos alunos do curso e participantes do estudo pode ser resumido da seguinte forma: a maioria eram mulheres (88\%) acima de 40 anos de idade (67\%); embora quase todos os participantes (91\%) tivessem um computador em casa, o acesso a Internet era baixo e apenas $37 \%$ acessavam mais de três vezes por semana; apenas $59 \%$ disseram ter acesso à Internet de banda larga em casa; e, a maioria (85\%) não tinha nenhuma experiência anterior com cursos via Internet (E-learning).

\subsection{Desenvolvimento da Pesquisa}

O curso foi dividido em dez disciplinas, onde uma parte do curso (20\%) ocorreu na forma tradicional face-a-face $(\mathrm{F} 2 \mathrm{~F})$, com encontros presenciais para apresentar o conteúdo em sala de aula. Nesses momentos, aconteciam apresentações, atividades práticas e avaliações escritas. A maior parte do curso (80\%) ocorreu via Internet, utilizando as ferramentas do Ambiente Virtual de Aprendizagem (AVA), tais como fórum, quiz e chat. Normalmente, uma disciplina durava cerca de dois meses e os alunos tinham de realizar, em média, três atividades virtuais. O AVA estava disponível 24 horas por dia, todos os dias da semana para os alunos poderem consultar informações ou realizar as atividades.

A tecnologia SMS foi escolhida para envio das mensagens para os estudantes devido às condições nas quais o curso aconteceu. Em muitas cidades no interior, onde a maioria dos alunos residia, não havia conexão de Internet fixa e estável. Além disso, as mensagens SMS estavam presentes em todos os celulares e não demandavam a instalação de aplicativos de terceiros. Desta forma, pretendemos envolver o máximo de alunos possível, contornando problemas técnicos ou financeiros que pudessem restringir o público participante. $\mathrm{O}$ uso de um aplicativo de mensagens implicaria na necessidade de uma equipe de suporte para solucionar problemas de instalação, de uso e até de Internet.

Para o estudo, os alunos foram divididos em dois grupos: A, grupo de controle, que era composto por 374 alunos que não receberam as mensagens SMS; B, grupo experimental, que contava com 100 alunos que receberam as mensagens em seus celulares. Em cada uma das turmas, 10 alunos foram selecionados aleatoriamente para formar o grupo B, aos quais foram enviados e-mails informando que estavam participando deste estudo e solicitando o consentimento deles. Durante o estudo, os estudantes de ambos os grupos mantinham a habitual interação entre eles.

A disciplina "Políticas Educacionais e Gestão Pedagógica" foi selecionada para possibilitar o estudo de caso com as mensagens SMS como suporte à aprendizagem. Três atividades, cada uma com uma pergunta, foram propostas para os alunos, as quais deveriam ser respondidas através do ambiente virtual na Internet. Essas atividades foram disponibilizadas no AVA para todos os alunos e enviadas via SMS apenas para 
aqueles do Grupo B. Todos os alunos precisavam respondê-las, podendo usar qualquer editor de texto e, posteriormente, enviar a resposta através do AVA.

$\mathrm{O}$ estudo de caso aconteceu em paralelo com a disciplina "Políticas Educacionais e Gestão Pedagógica", entre os meses de julho a setembro. Três mensagens de celular foram enviadas, cada uma com uma pergunta, em um intervalo de 10 dias entre elas. As mensagens móveis foram enviadas para os telefones celulares dos alunos, ao mesmo tempo em que a atividade tornou-se disponível no AVA. Para cada pergunta, os alunos tiveram um prazo de 10 dias para resolver e concluir a atividade. Porém, também foram aceitas respostas após o prazo.

Especificamente neste estudo, gostaríamos de saber se as mensagens SMS melhoravam a participação dos alunos no curso. Assim, buscamos responder as seguintes questões:

Q1: O uso de mensagens de celular incentivava os alunos a cumprirem as atividades atribuídas a eles?

Q2: O uso de mensagens de celular faz com que os alunos resolvam as questões mais rapidamente?

\section{RESULTADOS}

Esta seção apresenta os resultados obtidos com o estudo de caso, destacando-se a análise dos dados obtidos a partir das atividades realizadas pelos alunos de ambos os grupos (experimental e de controle) e a avaliação da satisfação deles com o uso da tecnologia das mensagens móveis, obtida a partir de um questionário aplicado.

\subsection{Análise dos dados}

Todos os dados utilizados neste estudo foram obtidos a partir dos registros contidos no AVA utilizado no curso $\left(M_{o o d l e}{ }^{1}\right)$. Esses registros permitem que consultas sejam feitas sobre, por exemplo, o momento em que as atividades foram iniciadas e o instante exato em que cada um dos alunos concluiu suas atividades. Para responder Q1 e Q2 e estatisticamente comprovar os resultados do estudo, foram usados os testes t-teste, z-teste e f-teste. Um dos pressupostos básicos para a realização das análises estatísticas é que as variáveis seguem Distribuição Normal. Para testar a hipótese nula de normalidade das variáveis analisadas no presente estudo foi utilizado o teste de Kolmogorov-Smirnov ${ }^{2}$, com base no qual se concluiu que, para um nível de significância de 5\%, a hipótese nula foi rejeitada.

Taxa de cumprimento. O primeiro ponto analisado foi a diferença entre as taxas médias de cumprimento das atividades realizadas pelos grupos experimental e de controle. Tentou-se identificar se o número de alunos que concluíram as atividades do grupo experimental foi maior do que o número daqueles que completaram as atividades do grupo de controle (Questão Q1). A importância desta análise é porque os alunos que não concluíram ficaram com notas ZERO nas atividades pendentes, o que impacta diretamente no desempenho global deles no curso.

Para analisar as diferenças entre as taxas médias de cumprimento, postulamos as seguintes hipóteses:

\footnotetext{
${ }^{1}$ https://moodle.org/

2 teste Kolgomorov-Smirnov é um teste não paramétrico de igualdade de distribuições subjacentes que pode ser usado para comparar duas amostras.
} 
H0: Não há nenhuma diferença entre as taxas de cumprimento entre os alunos dos grupos experimental e controle;

H1: A taxa média de cumprimento no grupo de controle é menor do que a do grupo experimental.

Levando-se em consideração as respostas das atividades dos alunos dos Grupos A e B, foi aplicado o z-test para medir as taxas de cumprimento, cujos valores são mostrados na Tabela 2. A partir desses resultados, pode-se concluir que, para uma significância de 5\%, a hipótese H0 é rejeitada. Portanto, a taxa média de conclusão das atividades do grupo de controle é menor do que a do grupo experimental (Hipótese H1).

Desta forma, a partir dos resultados obtidos com os testes estatísticos pode-se afirmar que, com 95\% de confiança, o grupo que recebeu as mensagens SMS teve melhor desempenho com relação ao cumprimento das atividades. Os valores apontam um percentual de conclusão das atividades entre 6,61\% e 19,49\% superior ao grupo que não recebeu as mensagens.

Tabela 2 - Resultados das taxas de cumprimento das atividades

\begin{tabular}{lrr}
\hline & Grupo A (Controle) & Grupo B (Experimental) \\
\hline Sucesso & 299 & 93 \\
Falha & 75 & 7 \\
Taxa de sucesso (\%) & 0,799 & 0,930 \\
\hline Valor-P: 0,0011 & \\
Estatística do teste Z: 3,065 & \\
Valor Crítico (0,05 uma cauda): 1,645 & \\
\hline Intervalo de Confiança: 95\% & Limite Superior: 0,1949 \\
\hline Limite Inferior: 0,0661 & \\
\hline
\end{tabular}

Tempo de resposta. Outro aspecto analisado foi o tempo que os alunos dos diferentes grupos levaram para completar as atividades. Procurou-se identificar se os alunos que receberam as mensagens SMS responderam em um intervalo de tempo menor que os estudantes que não receberam (Questão Q2). Esta análise é importante porque determinadas atividades podem demandar um curto espaço de tempo para serem resolvidas e, consequentemente, exigirem uma maior agilidade na resposta dos alunos.

Para analisar as diferenças entre tempos médios de resposta, colocamos as seguintes hipóteses:

H0: A diferença entre o tempo médio de resposta entre os estudantes dos grupos experimental e de controle é igual a zero.

H1: A diferença entre o tempo médio de resposta entre os estudantes dos grupos experimental e controle é diferente de zero.

Os resultados do t-teste para as diferenças no tempo de resposta médio, apresentados na Tabela 3, indicam que, para um nível de significância de 5\%, a hipótese H0 é rejeitada. Ou seja, pode-se concluir que, com um nível de confiança de 95\%, o tempo médio de resposta entre os grupos é diferente e os alunos no grupo que receberam as mensagens SMS responderam mais rapidamente, com tempos entre 1,97 e 78,9 horas mais rápidos. 
Tabela 3 - Resultado dos testes estatísticos para os tempos de conclusão das atividades

\begin{tabular}{lrr}
\hline Estatísticas descritivas & Grupo A (Controle) & Grupo B (Experimental) \\
\hline Média (horas) & 185,17 & 144,73 \\
Variância $\left(\sigma^{2}\right)$ & $32.089,11$ & $11.170,04$ \\
Tamanho da amostra (n) & 299 & 93 \\
\hline Intervalo de Confiança: 95\% & & \\
\hline Limite Inferior: 1,97 & Limite Superior: & 78,90 \\
\hline
\end{tabular}

\subsection{Avaliação dos participantes}

Para medir o nível de aceitação das mensagens móveis no curso, os alunos do grupo experimental receberam uma pesquisa on-line, depois de terem completado as suas atividades. A Tabela 4 resume as respostas ao questionário de avaliação. $\mathrm{O}$ objetivo foi determinar a opinião dos alunos sobre as atividades, se eles estavam satisfeitos com o uso de mensagens de celular e se eles gostariam de usar esta tecnologia ao longo do curso. Dos 100 estudantes do grupo experimental, 78 responderam anonimamente ao questionário de avaliação.

Os resultados desta avaliação são encorajadores. A maioria (94\%) dos estudantes afirma que as mensagens de celular ajudaram a completar as tarefas atribuídas. Entre outras respostas, quase todos os alunos que receberam as mensagens (92\%) disseram que gostariam que este recurso fosse usado em outras atividades do curso. Apenas 17\% dos alunos tiveram dificuldade para ler a pergunta que foi enviada para seu telefone celular.

Além das questões objetivas, o questionário tinha um campo em branco no qual os participantes do estudo puderam expressar suas opiniões sobre o uso de mensagens SMS no curso. A seguir, são apresentadas algumas dessas respostas:

"Já que não temos aqui na comunidade banda larga de Internet, receber as atividades pelo celular é muito bom, porque assim não tem como esquecer."

"Contribuirá em muito, especialmente quando no momento não for possível acessar o AVA. Como eu já sabia do que se tratava a pergunta não foi necessário consultar o ambiente para começar a respondê-la."

"Achei bem interessante a novidade. Confesso que me senti bastante motivada com o uso do telefone celular, pois posso estar sempre em dia com as atividades, visto que o celular está comigo 24 horas, diferente do computador."

"Nem sempre o tempo no trabalho contribui para acessar o ambiente virtual, então creio que as mensagens SMS sejam uma forma de reforçar o nosso compromisso."

Tabela 2 - Respostas do questionário de avaliação com os participantes.

\begin{tabular}{lc}
\hline \multicolumn{1}{c}{ Questões do questionário de avaliação } & Concorda (\%) \\
\hline Você concorda que o envio das mensagens foi positivo na execução das atividades? & 94 \\
Você sentiu-se mais incentivado a responder as perguntas após receber as mensagens & 85 \\
em seu celular? & 92 \\
Você gostaria que as mensagens SMS fossem usadas em outras atividades? & 67 \\
Você prefere receber as perguntas no celular ou acessá-las no AVA? & 17 \\
Você teve problemas para ler as perguntas recebidas no seu celular? & \\
\hline
\end{tabular}




\section{DISCUSSÃO}

A partir das respostas ao questionário de avaliação de satisfação com os participantes sobre o uso do SMS no curso, pode-se constatar que a maioria deles estava satisfeito e que apreciaram o uso dessa tecnologia. Os principais benefícios apontados foram: (1) não necessita que os alunos (que também são professores e estão ocupados durante todo o dia) entrem no AVA para visualizar as atividades atuais e (2) as mensagens de celular chegam onde os alunos estão e em qualquer hora, mesmo na ausência de acesso à Internet. Quando os alunos foram questionados se as mensagens SMS tiveram efeito positivo sobre o desempenho na disciplina, 94\% afirmaram que sim, que elas tinham influência positiva sobre a execução das atividades.

Além do questionário de avaliação, a partir da análise estatística realizada nesse estudo, pode-se constatar que os estudantes que receberam as mensagens de celular tiveram resultados melhores que aqueles que não receberam. $O$ grupo experimental teve melhores resultados tanto nas taxas de cumprimento das atividades quanto no tempo de resolução delas. Eles foram quase $20 \%$ mais eficazes na realização de suas atividades e até 78 horas mais rápidos do que os alunos do grupo que não recebeu as mensagens. A margem de confiança para estes resultados é de $95 \%$.

Neste artigo, as mensagens SMS foram enviadas em uma única direção: do curso para os estudantes. A principal razão para a escolha dessa abordagem foi devido aos custos que os alunos teriam que arcar se tivessem que enviar mensagens. Alguns estudos apontam a questão dos custos com o envio de mensagens como um impedimento para a utilização do SMS por estudantes (Petrova, 2010; Hayati et al,, 2013).

É importante destacar que os resultados apontados nesse estudo foram obtidos em um contexto onde a maioria dos alunos não tinha uma boa conexão de Internet. Além disso, todos eram professores de escolas públicas, com muitas atividades profissionais ao longo do dia. Assim, esses fatores podem ter favorecido os bons resultados obtidos no estudo. Desta forma, outras pesquisas são necessárias para ver se os benefícios apontados nesse estudo são obtidos em outros contextos.

\section{CONCLUSÃO}

Este estudo avaliou o uso de mensagens de celular em um curso de especialização semipresencial para professores da rede pública do Ceará. Concluiu-se que o uso desta tecnologia aumentou a participação dos alunos, como observado através de taxas de cumprimento, os tempos em que as atividades foram concluídas e as respostas ao questionário de avaliação aplicado.

Desta forma, ressalta-se que o m-learning baseado em mensagens de celular pode se tornar uma ferramenta importante em cursos semipresenciais. Considerando-se o alcance dos telefones celulares, benefícios do uso dessa solução podem ser percebidos mesmo em regiões economicamente desfavorecidas. Além disso, a partir do testemunho dos participantes, pode-se concluir que o serviço de mensagens SMS é uma alternativa relevante para o reforço de aprendizagem daqueles que trabalham o dia todo.

Por fim, destaca-se que o serviço SMS não precisa de internet. Isso aumenta o alcance da solução apresentada neste artigo, porque permite incluir alunos que não têm acesso fácil à Internet de banda larga, o que é uma realidade de diversas comunidades do interior. Soma-se a isso, o fato de tecnologias como $3 \mathrm{G}$ e $4 \mathrm{G}$ não chegarem a esses locais, dificultando o uso de aplicativos de mensagens como WhatsApp e Telegram. 


\section{REFERÊNCIAS}

ABU-AL-AISH, Ahmad; LOVE, Steve; HUNAITI, Ziad. Mathematics students' readiness for mobile learning. International Journal of Mobile and Blended Learning (IJMBL), v. 4, n. 4, p. 1-20, 2012.

BERE, Aaron. Toward assessing the impact of mobile security issues in pedagogical delivery: A mobile learning case study. In: Science and Information Conference (SAI), 2013. IEEE, 2013. p. 363-368.

BOTICKI, Ivica et al. Teaching and learning computer science sorting algorithms with mobile devices: A case study. Computer Applications in Engineering Education, v. 21, n. S1, p. E41-E50, 2013.

CHUANG, Yeu-Hui; TSAO, Chiung-Wen. Enhancing nursing students' medication knowledge: The effect of learning materials delivered by short message service. Computers \& Education, v. 61, p. 168-175, 2013.

CHURCH, Karen; DE OLIVEIRA, Rodrigo. What's up with whatsapp?: comparing mobile instant messaging behaviors with traditional SMS. In: Proceedings of the 15th International conference on Human-computer interaction with mobile devices and services. ACM, 2013. p. 352-361.

GASAYMEH, Al-Mothana M.; ALDALALAH, Osamah M. The Impact of Using SMS as Learning Support Tool on Students' Learning. International Education Studies, v. 6, n. 10, p. 112, 2013.

GUERENA E. Benefits of Text Messaging vs. Mobile Messaging Apps. Available in https://www.mobilecommons. com/blog/2014/09/benefits-text-messaging-vs-mobilemessaging-apps/. Accessed on July 30, 2016.

HAYATI, Abdolmajid; JALILIFAR, Alireza; MASHHADI, Amir. Using Short Message Service (SMS) to teach English idioms to EFL students. British Journal of Educational Technology, v. 44, n. 1, p. 66-81, 2013.

HWANG, Gwo-Jen; WU, Po-Han; KE, Hui-Ru. An interactive concept map approach to supporting mobile learning activities for natural science courses. Computers \& Education, v. 57, n. 4, p. 2272-2280, 2011.

KIM, Hyewon; LEE, Mi Young; KIM, Minjeong. Effects of Mobile Instant Messaging on Collaborative Learning Processes and Outcomes: The Case of South Korea. Educational Technology \& Society, v. 17, n. 2, p. 31-42, 2014.

MALCOLM R. Mobile Messaging Report - Global Insights Chat Apps and SMS Usage. Disponível em https://www.mblox.com/blog/2016/06/mobile-messaging-report-globalinsights-chat-apps-sms-usage/. Acesso em julho de 2016.

MARÇAL, Edgar; ANDRADE, Rossana; RIOS, Riverson. Aprendizagem utilizando dispositivos móveis com sistemas de realidade virtual. RENOTE, v. 3, n. 1, 2005.

MEF - Mobile Ecosystem Forum. Global Insights into Chat Apps and SMS Usage. Mobile Messaging Report, 2016.

PETROVA, Krassie. An implementation of an mLearning scenario using short text messaging: an analysis and evaluation. International Journal of Mobile Learning and Organisation, v. 4, n. 1, p. 83-97, 2009.

PLANA, Mar Gutiérrez-Colon et al. Improving learners' reading skills through instant short messages: A sample study using WhatsApp. Global perspectives on ComputerAssisted Language Learning. Glasgow, July. 2013. 